\title{
Preference for self-administration of a low dose of morphine into the ventral tegmental area rather than into the amygdala in mice
}

\author{
VINCENT DAVID and PIERRE CAZALA \\ Université de Bordeaux I, Talence, France
}

\begin{abstract}
BALB/c mice were bilaterally implanted with two guide cannulae, the tips of which were positioned either $1.5 \mathrm{~mm}$ above the amygdala (AMY) and the ventral tegmental area (VTA)(AMY-VTA subjects) or $1.5 \mathrm{~mm}$ above the AMY and $2.3 \mathrm{~mm}$ above the VTA (D.vta)(AMY-D.vta subjects). On each experimental day, a stainless steel injection cannula was inserted into each brain structure. The experiment was carried out in a Y-maze. During a preliminary phase, which lasted 4 days, animals were allowed to self-inject morphine successively into the AMY and into the VTA, or into the AMY and into the D.vta. From the 5th day, animals of each group were given the possibility of choosing between the two sites. Four subgroups were constituted depending on the dose of morphine used ( 5 and 50 ng: AMY5ng-VTA5ng, AMY50ng-VTA50ng, AMY5ng-D.vta5ng, and AMY5ng-D.vta50ng). The AMY5ng-VTA5ng group rapidly differentiated between the two injection sites and showed a marked preference for self-injection into the VTA. In the AMY50ng-VTA50ng group, no significant preference was observed, with the animals tending to alternate self-injection into the AMY and VTA. The AMY5ng-D.vta5ng group discriminated between the two sites and self-injected morphine preferentially into the AMY. The discrimination performance of the AMY5ng-D.vta50ng group was not statistically different from that at chance level. These results demonstrate that mice are capable of discriminating, at the intracerebral level, the motivational or rewarding components of morphine when the dose available is low $(5 \mathrm{ng})$. The preference manifested is highly influenced by the location of injection cannulae. The positive effect of a low dose of morphine appeared stronger in the VTA than in the AMY. However, the location of injection cannulae $0.8 \mathrm{~mm}$ above the VTA induced a marked preference for self-injection into the AMY. Consequently, the rewarding effects of morphine into the VTA probably results from a local action of the drug and not from a dorsal diffusion.
\end{abstract}

The optimal experimental method to demonstrate that a given drug produces its rewarding action via the mediation of a particular central receptor field is to show that this drug is directly self-administered into that brain region. Thus self-injection of morphine by the rat has been reported for a large number of brain sites, including the ventral tegmental area (VTA), preoptic area, septum and lateral hypothalamus (Bozarth \& Wise, 1980, 1981; Olds, 1979; Stein \& Olds, 1977; Welzl, Kuhn, \& Huston, 1989). The discovery of a large number of sites supporting a finite intracranial self-administration (or similarly, self-stimulation) has argued for the existence of multiple brain reward systems (Phillips, 1984; Wise \& Bozarth, 1984). However, in spite of this, we do not know if reward elicited by a given dose of drug injected into one site is more intense than that produced by injection into another brain site.

Previous studies from our laboratory have clearly demonstrated the existence of a structure-related differentia-

This investigation was supported by the CNRS (URA 339). We would like to thank T. Durkin for correcting the English text and J. Ducout and L. Decorte for technical assistance. Correspondence should be addressed to P. Cazala, Laboratoire de Neurosciences Comportementales et Cognitives, CNRS URA 339, Université de Bordeaux I, Avenue des Facultés, 33405 Talence Cedex, France. tion related to the self-administration of morphine into the lateral and medial parts of the hypothalamus, mesencephalic central gray area, amygdala (AMY), VTA, and ventral part of the reticular formation in BALB/c mice (Cazala, 1990; Cazala, Darracq, \& Saint-Marc, 1987; David \& Cazala, 1994a, 1994b). We found that, for a given dose of the drug, the characteristics of the self-administration response markedly varied according to the brain structure considered, suggesting that a given dose of morphine elicits a reward component that differs quantitatively and/ or qualitatively as a function of the injection site.

However, such differences in reward intensity as measured operationally by acquisition rate could also be due to memory-improving effects or involve opiate modulation of more general processes like attention and stimulus association. Thus, we cannot conclude unequivocally that the affective value of the stimulus is solely responsible for the phenomenon of the influence of site dependence on the rate of acquisition of self-administration. In operational terms, we may posit the following: Is an animal able to differentiate, in a choice situation, the drug effects obtained by local injections of a given dose of drug into two different brain sites? In order to attempt an answer to this question, animals implanted with two guide cannulae were placed in an experimental situation offering 
the possibility of choosing between self-injection of morphine into the AMY or the VTA; both structures contain a high density of $\mu$ receptors (Mansour, Fox, Akil, \& Watson, 1995) for which morphine has high affinity (Simantov, Childers, \& Snyder, 1978), and both are sites from which a regular self-administration response for this drug has been observed (David \& Cazala, 1994a). Moreover, even though previously we have studied the extent of diffusion of drug in our protocol by varying the cannula placements (David \& Cazala, 1994b), in the present experiment, in order to ensure that dorsal diffusion (the most important component, according to Wise \& Hoffman, 1992) of the injected substance was not responsible for the observed effects, we compared the behavior of the experimental animals with that of an additional independent group of subjects having injection cannulae located $0.8 \mathrm{~mm}$ above the VTA.

\section{METHOD}

\section{Animals and Surgery}

In the present experiment we used 23 male mice of the BALB/c By Jico strain (Iffa-Credo). At 9 weeks of age, they were housed individually with ad-lib access to food and water in a temperaturecontrolled room $\left(23^{\circ} \mathrm{C}\right)$ with a $12: 12$-h light:dark cycle (light on at $08.00 \mathrm{~h}$ ). The animals were 11-12 weeks old (body weight, 27$30 \mathrm{~g}$ ) at the beginning of the experiment. Under deep sodium thiopental anesthesia $(90 \mathrm{mg} / \mathrm{kg})$ the animals were bilaterally implanted with guide-cannulae (outer diameter, $0.460 \mathrm{~mm}$; inner diameter, $0.255 \mathrm{~mm}$ ) the tips of which were positioned either $1.5 \mathrm{~mm}$ above the AMY and VTA $(n=15)$ or respectively $1.5 \mathrm{~mm}$ above the AMY and $2.3 \mathrm{~mm}$ above the VTA (D.vta, $n=8$ ). The stereotaxic coordinates used were the following: AMY, $1.20 \mathrm{~mm}$ posterior to the bregma, $\pm 3.00 \mathrm{~mm}$ lateral to the sagittal line, and $3.40 \mathrm{~mm}$ vertically below the surface of the skull; VTA, anteroposterior distance (ap) referring to interaural line $+0.40 \mathrm{~mm}$, lateral (l) referring to sagittal line $\pm 0.20 \mathrm{~mm}$; vertical (v) from the surface of the skull $+3.30 \mathrm{~mm}$; D.vta, ap $+0.40 \mathrm{~mm}, 1 \pm 0.20 \mathrm{~mm}$, and $v+2.50 \mathrm{~mm}$. The incisor bar was level with the interaural line. Mice were allowed to recover from the operation for 1 week.

\section{Materials and Experimental Protocol}

Self-injection procedure. On each experimental day, two stainless steel injection cannulae (o.d. $0.229 \mathrm{~mm}$; i.d. $0.127 \mathrm{~mm}$ ) were inserted in the injection sites and were held fixed in position by means of a small connector. Injection cannulae were connected by flexible polyethylene tubings to two independent micro-injection systems that each housed a 5- $\mu 1$ Hamilton syringe. The tips of the injection cannulae projected beyond the guide-cannulae by $1.5 \mathrm{~mm}$. By interrupting photo cell beams in the Y-maze (see behavioral procedure) mice could obtain the reinforcement (injection of morphine sulfate dissolved in Ringer's solution). Each self-injection $(50 \mathrm{nl})$ lasted $4 \mathrm{sec}$ (normal drug flow was controlled visually before and after injection of each animal). The least movement of the animal in the Y-maze was detected by an optical system. This information was transmitted to a microcomputer that commanded the rotation of the two injectors in the same direction as the animal's movement. This process avoided the rolling-up of the two flexible tubings; consequently, self-administration could be studied in freely moving mice (Cazala et al., 1987).

Behavioral procedure. Self-administration behavior was studied in an opaque Plexiglas Y-maze, the two arms of which were separated by a $90^{\circ}$ angle. The stem and arms were $31 \mathrm{~cm}$ long, $8 \mathrm{~cm}$ wide, and $12 \mathrm{~cm}$ high. The starting box $(14 \times 8 \mathrm{~cm})$ was separated from the stem by a horizontal sliding door. Horizontal sliding doors were also located at the entrance of each arm. A photo-electric cell was situated $6 \mathrm{~cm}$ from the end of each arm.

After a first habituation session during which no injection was delivered, the animals were submitted to two successive experimental phases. During a preliminary phase, to begin a trial, a mouse was placed in the starting box and after $1 \mathrm{~min}$ the door to the stem was opened. During the 1 st day, in each group (see below) a certain number of animals had to learn to trigger the injection of morphine by interrupting the photo-cell beam situated in the right arm (the left arm being closed), whereas the others had to go to the left arm (the right arm being closed). When the injection was terminated, the mouse was replaced into the starting box where it was retained for $1 \mathrm{~min}$, after which the door was reopened to begin a new trial. This phase lasted 4 days. An animal could obtain delivery of morphine into the AMY during the 1st and the 3rd days (in the right arm, e.g.; only one of the two cannulae being active) and delivery of morphine into the VTA during the 2 nd and the 4th days (in the left arm, e.g.; only the other cannula being active). For another subject morphine could be delivered into the AMY by entering in the left arm and into the VTA by entering in the right arm. The brain side chosen for injection into the AMY or VTA was different from 1 animal to another. The order of presentation of injections into the AMY and VTA was also different from 1 subject to another (during the 4 successive days, e.g., the order for an animal was AMY, VTA, AMY, VTA or VTA, AMY, VTA, AMY). Each daily session was composed of 10 trials. The same behavioral procedure was used for animals that self-injected morphine into the AMY or D.vta.

Four groups of subjects were constituted, two groups of AMYVTA and two groups of AMY-D.vta. In the first AMY-VTA group, the experiment was performed using the 5-ng dose of morphine for each structure $(n=6)$ and in the second group using the 50-ng dose of morphine for each structure $(n=9)$. In the first AMY-D.vta group, the experiment was performed using the 5-ng dose of morphine for each structure $(n=4)$ and in the second group using the 5-ng dose of morphine for the AMY and the 50-ng dose for the D.vta $(n=4)$, respectively.

The second experimental phase began on the 5th day. During this phase, the two arms of the Y-maze were opened and the animals were thus able to choose freely the brain structure into which they could self-inject morphine (the two cannulae being active). This phase lasted 6 days in the two AMY-VTA groups, 7 days in the AMY5ng-D.vta5ng group, and 9 days in the AMY5ng-D.vta50ng group. For the AMY5ng-VTASng group, the acquisition phase was followed by a reversal phase during which the arm allowing the self-injection of morphine into the AMY was permuted with the arm allowing self-injection of morphine into the VTA (7 days). Automatic equipment, triggered by opening the door of the startbox to begin a trial, recorded the latency to trigger each injection during the two phases.

\section{Histology}

At the end of the experiment, the animals were sacrificed with an overdose of thiopental. The head with the guide cannulae attached was placed in $10 \%$ formol for $72 \mathrm{~h}$. The guide cannulae were then withdrawn, and the brain was dissected and placed in a solution of formol containing $30 \%$ sucrose for another week. Frozen brains were then cut in a microtome to provide $60-\mu \mathrm{m}$ sections that were stained with $0.1 \%$ thionin to identify the injection site.

\section{Statistical Analyses}

Evolution of preference in each group (e.g., the AMY 5ngVTA5ng group) and latency to trigger the injection in each site (e.g., AMY5ng) were analyzed with one-way analyses of variance (ANOVAs) with repeated measures. Paired Student $t$ tests were used to compare the latencies of self-injections into each brain site during preliminary phase in the same group (e.g., latency of intraAMY vs. intra-VTA self-injection in the AMY5ng-VTA5ng group) 
and unpaired Student $t$ tests for comparison between groups. Student $t$ tests were also used to compare the mean of a punctual session choice with that at chance level (Winer, 1971).

\section{RESULTS}

\section{Discrimination Between the AMY and VTA}

During the preliminary phase (alternated self-injection in the two brain structures), we observed that in the AMY5ngVTA5ng group, the latency to trigger the injection into the VTA was shorter than that to trigger the injection into the AMY $[t(5)=3.68, p<.02$; Table 1]. In the AMY50ngVTA50ng group, the inverse tendency was recorded. Indeed, whereas the values of injection latency remained stable into the AMY, they markedly increased for injections into the VTA $[t(13)=2.53, p<.05$; Table 1].

During the choice phase, the AMY5ng-VTA5ng group rapidly differentiated between the two injection sites. This discrimination appeared from the first acquisition session $[t(5)=6.32, p<.01]$. During the following sessions, the discrimination performance increased further and the subjects of this group entered more and more frequently into the arm reinforced by the injection into the VTA $[F(5,25)=6.14, p<.001$; Figure 1].

Starting at the seventh session, the arm reinforced by injection into the VTA was permuted with the arm reinforced by injection into the AMY and the subjects were submitted to a reversal task. Despite the difficulty of this new condition, the subjects retained their previous choice of brain site, and from the third reversal session, the mean number of self-injections into the VTA was statistically different from that at chance level $[t(5)=3.32, p<$ $.05]$. The discrimination performance increased during the following sessions, $[F(6,30)=32.96, p<.001]$, and during the last reversal session was identical to that recorded at the end of the acquisition phase.

In the AMY50ng-VTA50ng group, no preference was observed between the two injection sites. The animals performed at random, which accounts for the fact that no significant progression of the mean level of discrimination performance was observed during the six successive sessions $[F(5,40)=0.26$, n.s. $]$.

Table 1

Mean Values (in Seconds) of the Latency ( $(S E M)$ to Trigger the Injection of Morphine Into the AMY, D.vta, and VTA, Recorded During the Preliminary Phase in the Different Groups Studied

\begin{tabular}{cll}
\hline Group & \multicolumn{2}{c}{ Latencies } \\
\hline AMY5ng-VTA5ng & AMY & $55.6 \pm 7.3$ \\
& VTA & $19.7 \pm 2.8$ \\
AMY50ng-VTA50ng & AMY & $44.4 \pm 16.3$ \\
& VTA & $62.9 \pm 13.6$ \\
AMY5ng-D.vta5ng & AMY & $47.2 \pm 8.4$ \\
& D.vta & $88.3 \pm 22.7$ \\
AMY5ng-D.vta50ng & AMY & $174.4 \pm 53.6$ \\
& D.vta & $228.1 \pm 44.3$ \\
\hline
\end{tabular}

Note-AMY, amygdala; VTA, ventral tegmental area; D.vta, $0.8 \mathrm{~mm}$ above the VTA.

\section{Discrimination Between the AMY and D.vta}

During the preliminary phase (alternated self-injection into each brain structure) in the AMY5ng-D.vta5ng group, the latency to self-inject morphine into the D.vta tended to be longer than that to trigger the injection into the AMY, although the difference observed was not statistically significant (Table 1). Latencies to self-inject morphine into the D.vta in this group were significantly longer than those to trigger the injection into the VTA in the AMY5ng-VTA5ng group $[t(8)=3.74, p<.01]$.

In the AMY5ng-D.vta50ng group, a marked increase of injection latencies was observed in the two structures by a statistical comparison with the values recorded in the AMY5ng-D.vta5ng group [AMY, $t(6)=2.34, p=.05$; D.vta, $t(6)=2.80, p<.05$; Table 1].

During the choice phase, the AMY5ng-D.vta5ng subjects differentiated between the two injection sites. During the seven successive sessions, these animals entered more and more frequently into the arm reinforced by the injection into the AMY $[F(6,18)=11.02, p<.001$; Figure 2].

In the AMY5ng-D.vta50ng group, 2 animals chose to self-inject morphine into the AMY, another chose selfinjection into the D.vta, and the last mouse performed at random. Thus the mean discrimination performance in this group was not statistically different from chance level over the nine successive sessions $[F(8,24)=0.30$, n.s.].

\section{Histology}

Injection sites were precisely located by following the track of each injection cannula. Figure 3 summarizes these placements into the AMY, VTA, and $0.8 \mathrm{~mm}$ above the VTA (D.vta). In the latter case, cannulae tips were located in the immediate proximity of the ventral part of the mesencephalic central gray area (CG), except for one that was situated more ventrally near to the VTA.

\section{DISCUSSION}

\section{Discrimination Between Reward Elicited From VTA and AMY: Low Dose of Morphine}

Our results demonstrate clearly that $\mathrm{BALB} / \mathrm{c}$ mice are able to differentiate between the rewarding effects of morphine injected into two different brain regions (VTA or AMY) during self-administration behavior when the dose delivered is low ( $5 \mathrm{ng}$ ). The marked preference for self-injection of a low dose of morphine into the VTA rather than into the AMY (AMY5ng-VTA5ng group) is probably due to the strong motivational and/or rewarding effects of this substance when injected into this mesencephalic structure. Moreover, the complete reversal observed in this group when the rewarded arms were inversed also attests to the clear differentiation between the affective states produced. This rewarding effect of morphine when injected into the VTA has previously been reported using two different techniques: conditioned 


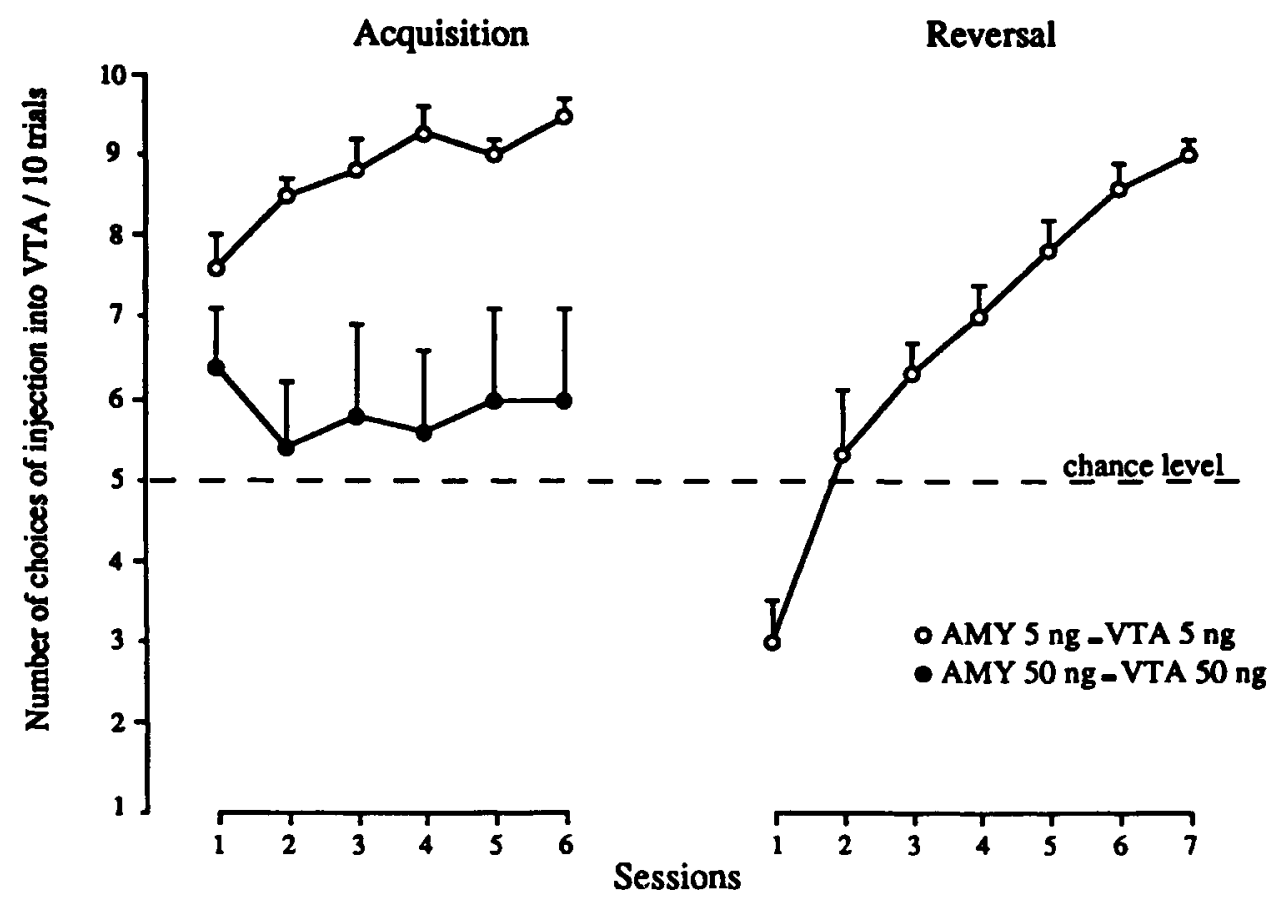

Figure 1. Mean number of self-injections of morphine $( \pm S E M)$ into the ventral tegmental area (VTA) recorded in the AMY5ng-VTA5ng and AMY50ng-VTA50ng groups during the "choice phase" of the spatial discrimination task.

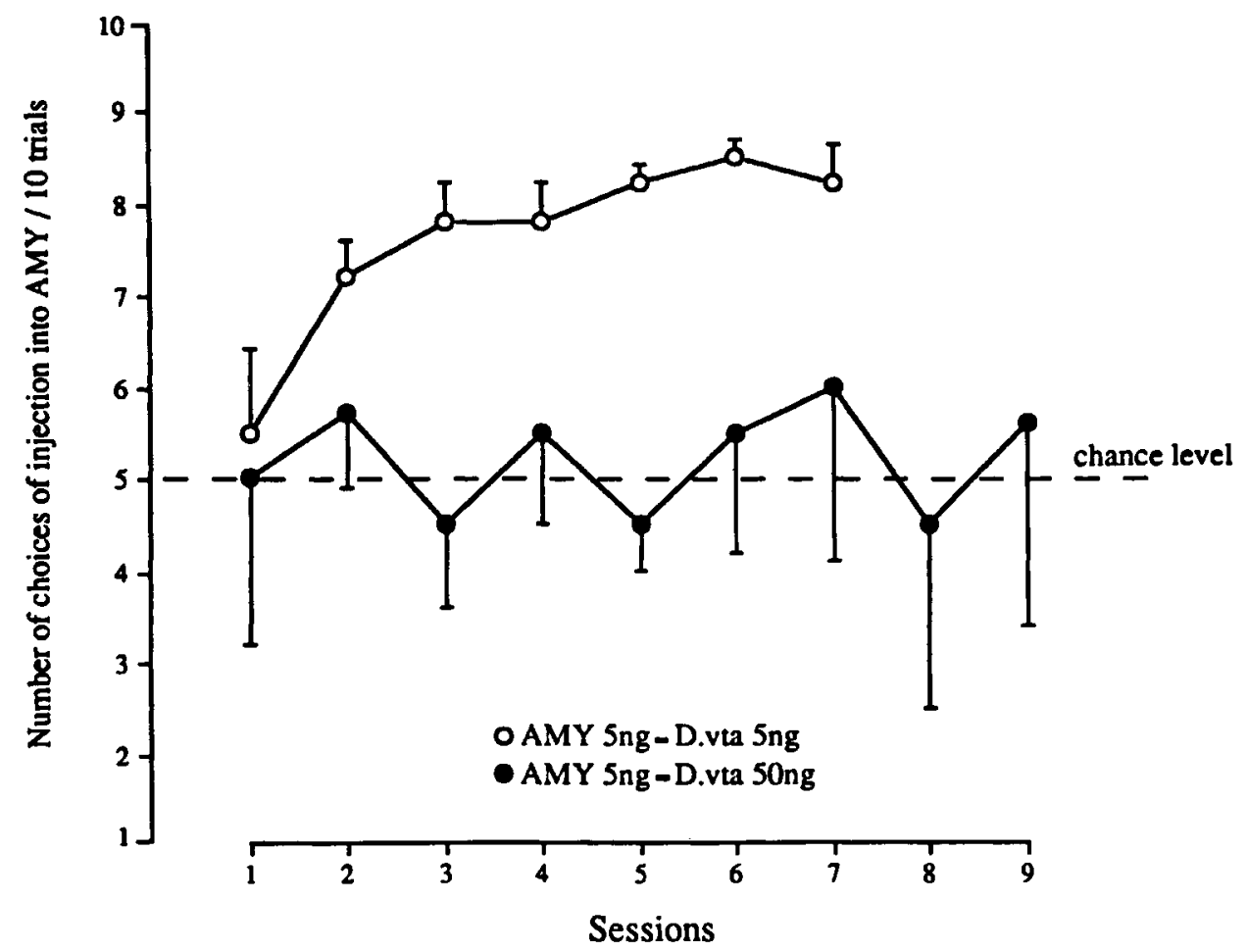

Figure 2. Mean number of self-injections of morphine ( $\pm S E M$ ) into the amygdala (AMY) recorded in the AMY5ng-D.vta5ng and AMY5ng-D.vta50ng groups during the "choice phase" of the spatial discrimination task. 


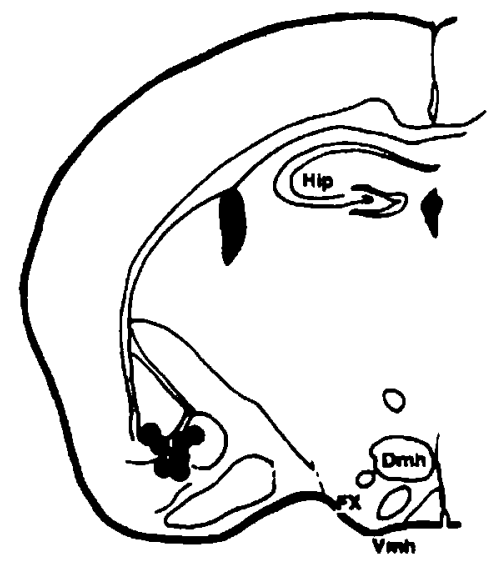

$2900 \mu$

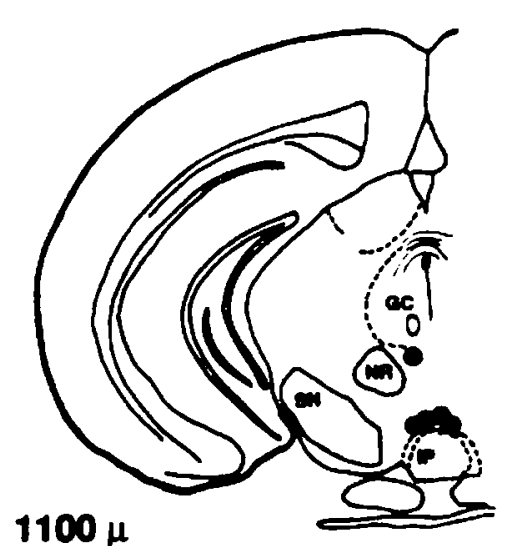

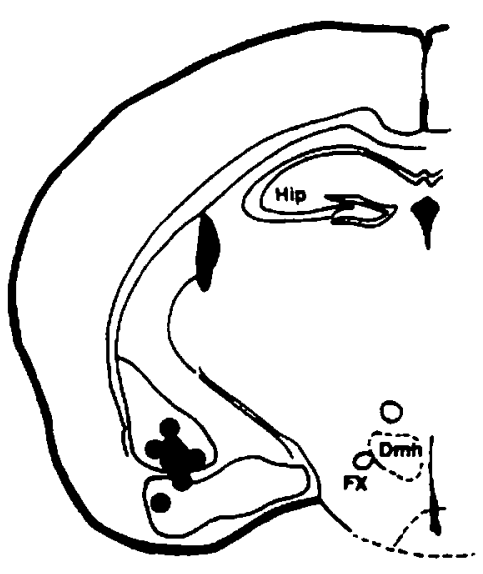

$2750 \mu$

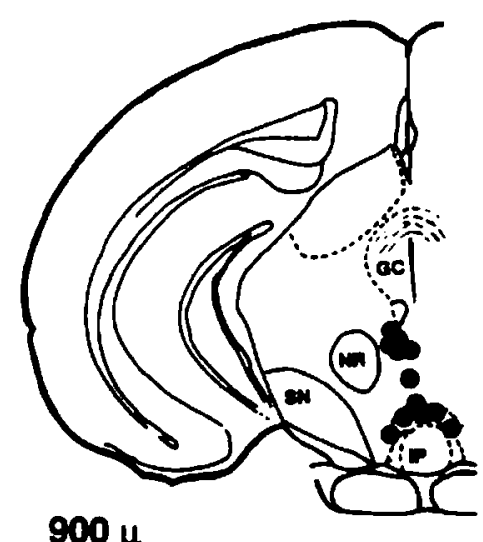

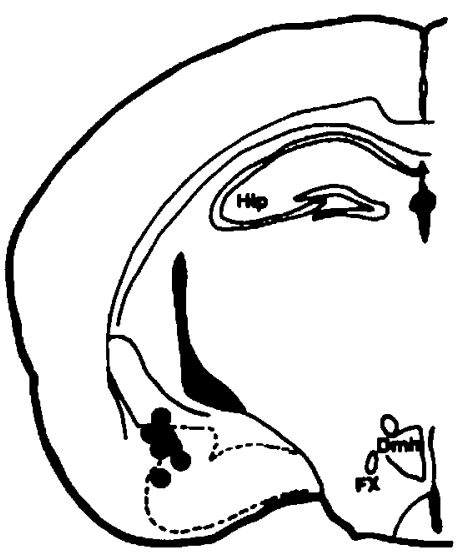

$2500 \mu$

Figure 3. The placements of the injection sites in the amygdala (AMM), in the ventral tegmental area (VTA), or in the D.vta are plotted on frontal section diagrams. The values in microns $(\mu)$ indicate the distance of the section from the frontal plane zero situated 1 mm posterior to the interaural line. (From Atlas stéréotaxique du cerveau de la souris (plates 4-6, 17, and 19-20), by A. Lehmann, 1974, Paris: Editions du Centre National de la Recherche Scientifique. Copyright 1974 by Centre National de la Recherche Scientifique. Adapted with permission). Dmh, nucleus dorsomedialis hypothalami; Fx, fornix; GC, substantia grisea centralis; Hip, hippocampus; IP, nucleus interpeduncularis; NR, nucleus ruber; NIII, nucleus nervi oculomotorii; SN, substantia nigra; Vmh, nucleus ventromedialis hypothalami.

place preference (Bozarth, 1987a; Phillips \& Le Piane, 1980 ) and intracranial self-administration in rats (Bozarth \& Wise, 1981; Welzl et al., 1989) and in mice (David \& Cazala, 1994a). Although drug preference experiments have previously been conducted using an intravenous self-injection protocol in monkeys (Deneau, Yanagita, \& Seevers, 1969), the present study is the first experiment, to our knowledge, that allows animals to freely choose which one of the two intracranial injection sites they prefer. The injection latencies, which are shorter in the case of the VTA than in the case of the AMY (recorded in the AMY5ng-VTA5ng group), confirm the stronger motivational effects of opiate injections into the VTA. Indeed, it seems impossible to explain such a marked choice preference simply via a facilitatory effect of morphine injections on sensorimotor parameters. Consequently, the intensity or quality of reward elicited by intra-VTA self-injection of this low dose of morphine appears to be greater than that elicited from the AMY.
According to available electrophysiological, biochemical, and behavioral data, opiate reward in the VTA is dependent on the activation of a dopaminergic neuronal mechanism. Stimulation of $\mu$-opioid receptors in vivo increases the firing rates of dopamine neurons in the VTA (Gysling \& Wang, 1983; Matthews \& German, 1984). Injection of opioid peptides into the VTA increases dopamine turnover in the terminal fields of dopaminergic VTA neurons (Cador, Rivet, Kelley, Le Moal, \& Stinus, 1989) and dopamine-depleting lesions of the VTA disrupt the acquisition of intravenous heroin self-injection (Bozarth, 1987b). Moreover, $\mu$-opioid activation also inhibits the firing frequency of nondopamine cells in the VTA (Gysling \& Wang, 1983), and unilateral lesion of VTA DA pericarya (6-OHDA) does not significantly change the number of $\mu$ opioid receptors present in this brain area (Dilts \& Kalivas, 1989). These data suggest that the majority of $\mu$ opiate receptors are located on nondopaminergic neurons intrinsic to the VTA. The stimulant ef- 
fects of $\mu$-opioids on dopamine cells would, thus, result indirectly (disinhibition) from a $\mu$-opioid-mediated inhibition of GABAergic interneurons on which $\mu$ receptors seem to be located (Kalivas, 1993).

However, it may be recalled that, for example, the nucleus accumbens seems to be another important site involved in opiate reward, a phenomenon that is somewhat less dopamine dependent or even nondopamine dependent (Koob \& Goeders 1989; Pettit, Ettenberg, Bloom, \& Koob 1984; Stinus, Cador, \& Le Moal, 1992). In any case, the major result of the present experiment indicates unequivocally that, in a low-dose situation, animals are able to differentiate between both affective states produced and to choose which one they prefer. Using this type of within-subject choice protocol, further dose-effect experiments will be able to clarify this apparent superiority of the VTA (in a low-dose situation) over the AMY - or other putative brain regions-in mediating opiate reward.

\section{Discrimination Between Reward Elicited From the VTA and AMY: High Dose of Morphine}

When the dose of morphine available was ten times higher (AMY50ng-VTA50ng group), the animals no longer chose between the two self-injection sites. Two main reasons can be invoked to explain this phenomenon. On the one hand, it is possible that animals do not differentiate clearly between the effects of morphine injected into the AMY and the VTA at this high dose. Thus, the ability to express any preference between sites becomes impossible for them. Indeed, if the existence of preference implies a differentiation by the animals between the two affective states produced by the drug injected into either the AMY or the VTA, the absence of preference does not necessarily indicate that the two affective states produced are identical. This may explain our observations of differences in injection latencies when no arm preference was apparent. On the other hand, it is also possible that eventual aversive effects of morphine (Grabowsky \& Cherek, 1983) produced at the 50ng dose may disturb preference for self-administration into the VTA. In previous experiments, we reported that in independent groups the acquisition rate recorded from VTA-injected animals was more rapid with the dose of $5 \mathrm{ng}$ of morphine than with the dose of $50 \mathrm{ng}$, whereas for the AMY group the acquisition rates were similar with these two doses (David \& Cazala, 1994a). In accordance with these previous findings, we observed, during the preliminary phase of the present experiment, a marked and progressive increase of the injection latency into the VTA (in the AMY50ng-VTA50ng group), whereas in the case of the AMY, injection latency was similar in both groups. Such observations allow two different interpretations. First, aversive emotional reaction to the drug at this high dose could be stronger from injections into the VTA than into the AMY. Second, it is well known that, in self-administration protocols, a direct relationship can be observed between the dose of drug injected and the length of the time interval between successive injections
(Koob, Vaccarino, Amalric, \& Bloom, 1987). Thus, a rapid summation of the hedonic effects of successive injections is probably responsible for the lengthening of the injection delays observed in the case of the VTA. Consequently, the animals probably prefer to alternate self-injections into the VTA and the AMY.

\section{Discrimination Between Morphine Injections Into the AMY and D.vta: Anatomical Specificity}

One important problem raised by the intracranial selfadministration paradigm concerns the anatomical specificity and the spatial delimitation of the reinforcing effect produced by the substance injected (Bozarth, 1983). Is the effect produced due to the activation of receptors located in the vicinity close to the injection site or due to the involvement of more distant receptors by diffusion? We have previously shown that a $0.6-\mathrm{mm}$ lateral change in stereotaxic coordinates allows us to observe behavioral differences as clear as the presence or lack of drugseeking behavior (David \& Cazala, 1994b). However, the possibility of a dorsal efflux from the tip of the injection cannula may be envisaged (Wise \& Hoffman, 1992), especially in the case of a small brain structure such as the VTA. A clear answer to this possibility is provided by the fact that when the mesencephalic injection cannula was located $0.8 \mathrm{~mm}$ above the VTA, all the animals in the AMY 5ng-D.vta5ng group triggered injection of morphine into the AMY, with this choice being almost exclusive. This result is of major importance for the three following reasons: (1) It demonstrates that, in the AMY5ngVTA5ng group, the preference for self-injection into the VTA was effectively induced by the activation of opiate receptors located in, or very close to, this structure; (2) it confirms that a self-injection response can be induced from the AMY with a 5-ng dose of morphine (David \& Cazala, 1994a); and (3) it suggests that, at this low dose, the drug has no reinforcing effect in the brain area situated $0.8 \mathrm{~mm}$ above the VTA. This conclusion also appears to be confirmed by the fact that, during the preliminary phase, the latencies of self-injection tended to be longer for D.vta placements than for AMY placements.

When the dose of morphine injected into the D.vta was 10 times higher than that injected into the AMY (AMY5ng-D.vta50ng group), two phenomena were observed. First, only 1 animal among the 4 subjects composing this group showed a marked preference for selfinjection into the D.vta during the free-choice phase. Since histological control revealed that the subject choosing to self-inject morphine into the D.vta was the one having the tip of injection cannula located nearest to the VTA, possible activation of this structure by the 50-ng dose of morphine may have influenced the animal's choice. Second, during the preliminary phase, we noted, for the two structures, that the injection latencies markedly increased. This increase was particularly large in the case of D.vta, which suggests the possibility that aversive effects of high doses of morphine injected in this brain area constrain the subjects to space out injection 
frequency. An analysis of the consequences of morphine injections suggests that this drug, which has strong reinforcing properties, may also have aversive effects in certain circumstances (Grabowsky \& Cherek, 1983). The rewarding effects of morphine are predominantly associated with activation of $\mu$ receptors, for which it has high affinity (Simantov et al., 1978). On the other hand, the aversive consequences of opiates are associated with the activation of $\kappa$ receptors peripherally (Bechara \& Van der Kooy, 1987) as well as centrally (Bals-Kubik, Ableitner, Herz, \& Shippenberg, 1993). The very low density of $\mu$ receptors in the area situated between ventral CG and VTA (Atweh, 1983) could explain the very weak reinforcing effects of morphine injected in this region. The presence of $\kappa$ receptors in the CG (Mansour et al., 1995) and, therefore, in the immediate proximity of our injection sites in the D.vta could underlie the origin of the aversive effect of morphine, which, although being a preferential $\mu$-agonist, is not exclusively so (Robson, Peterson, \& Kosterlitz, 1983), especially when a high dose is applied. The concomitant lengthening of injection latencies into the AMY can probably be explained by a temporary loss of motivation, which is a consequence of aversive effects induced by morphine when injected into the D.vta. This phenomenon, however, progressively diminishes since 2 mice of this group subsequently selfadministered the 5-ng dose of morphine into the AMY during the choice phase.

In conclusion, the major results of our study can be summarized as follows. A within-subject discrimination between the different affective states produced by injections of morphine into either the AMY or the VTA can be observed with a low dose of the drug. In this freechoice situation, BALB/c mice preferentially choose to self-inject the drug into the VTA. Whereas self-injection of low doses of morphine into either the VTA or AMY is observed to be rewarding in both structures (David \& Cazala, 1994a), our present within-subject experiment investigating site preference shows that the interoceptive reward value elicited from each site is quantitatively and/or qualitatively different. For two reasons, we may conclude that the drug effects observed in the present experiment are predominantly occurring in, or very close to, the injection sites: (1) The use of a low dose minimizes any eventual diffusion problems, and (2) (and more importantly), the dorsal displacement of VTA cannulae induces a complete inversal of choice from one site to the other.

\section{REFERENCES}

ATWEH, S. F. (1983). Characterization and distribution of brain opiate receptors and endogenous opioid peptides. In J. E. Smith \& J. D. Lane (Eds.), The neurobiology of opiate reward processes (pp. 5988). Amsterdam: Elsevier.

Bals-Kubik, R., Ableitner, A., Herz, A., \& Shippenberg, S. (1993). Neuroanatomical sites mediating the motivational effects of opioids as mapped by the conditioned place preference paradigm in rats. Journal of Pharmacology \& Experimental Therapeutics, 264, 489-495.

Bechara, A., \& VAN Der KoOY, D. (1987). Kappa receptors mediate the peripheral aversive effects of opiates. Pharmacology, Biochemistry \& Behavior, 28, 227-233.

BozARTH, M. A. (1983). Opiate reward mechanisms mapped by intracranial self-administration. In J. E. Smith \& J. D. Lane (Eds.), The neurobiology of opiate reward processes (pp. 331-359). Amsterdam: Elsevier.

Bozarth, M. A. (1987a). Neuroanatomical boundaries of the reward relevant opiate receptors field in the ventral tegmental area as mapped by the conditioned place preference method in rats. Brain Research, 414, 77-84.

BozARTH, M. A. (1987b). Ventral tegmental reward system. In J. Engel \& L. Oreland (Eds.), Brain reward system and abuse (pp. 1-17). New York: Raven Press.

BozARTH, M. A., \& WISE, R. A. (1980). Intracranial self-administration of morphine into various brain regions. Society for Neuroscience Abstracts, 6, 309.

BozARTH, M. A., \& WISE, R. A. (1981). Intracranial self-administration of morphine into the ventral tegmental area in rats. Life Sciences, 28, 551-555.

Cador, M., Rivet, J. M., Kelley, A. M., Le Moal, M., \& Stinus, L. (1989). Substance P, neurotensin and enkephalin injections into the ventral tegmental area: Comparative study on dopamine turnover in several forebrain structures. Brain Research, 486, 357-363.

CAZALA, P. (1990). Dose-dependent effects of morphine differentiate self-administration elicited from lateral hypothalamus and mesencephalic central gray area in mice. Brain Research, 527, 280-285.

Cazala, P., Darrace, C., \& Saint-Marc, M. (1987). Self-administration of morphine into the lateral hypothalamus in the mouse. Brain Research, 416, 283-288.

DAVID, V., \& CAZAla, P. (1994a). A comparative study of self-administration of morphine into the amygdala and the ventral tegmental area in mice. Behavioural Brain Research, 65, 205-211.

David, V., \& Cazala, P. (1994b). Differentiation of intracranial morphine self-administration behavior among five brain regions in mice. Pharmacology, Biochemistry \& Behavior, 48, 625-633.

Deneau, G., Yanagita, T., \& Seevers, M. H. (1969). Self-administration of psychoactive substances by the monkey: A measure of psychological dependence. Psychopharmacologia, 16, 30-48.

DiLTS, R. P., \& Kalivas, P. W. (1989). Autoradiographic localization of $\mu$-opioid and neurotensin receptors within the mesolimbic dopamine system. Brain Research, 488, 311-327.

Grabowski, J., \& Cherek, D. R. (1983). Conditioning factors in opiate dependence. In J. E. Smith \& J. D. Lane (Eds.), The neurobiology of opiate reward processes (pp. 175-210). Amsterdam: Elsevier.

Gysling, K., \& WANG, R. Y. (1983). Morphine-induced activation of A10 dopamine neurons in the rat. Brain Research, 277, 119-127.

KaLIVAS, P. W. (1993). Neurotransmitter regulation of dopamine neurons in the ventral tegmental area. Brain Research Review, 18, 75113.

КоOв, G. F., \& GoEders, N. E. (1989). Neuroanatomical substrates of drug self-administration. In J. M. Liebman \& S. J. Cooper (Eds.), Neuropharmacological basis of reward (pp. 214-263). Oxford: Oxford University Press.

Koob, G. F., Vaccarino, F., Amalric, M., \& Bloom, F. E. (1987). Positive reinforcement properties of drugs: Search for neural substrates. In J. Engel \& L. Oreland (Eds.), Brain reward systems and abuse (pp. 35-50). New York: Raven Press.

LEHMANN, A. (1974). Atlas stéréotaxique du cerveau de la souris. Paris: Editions du Centre National de la Recherche Scientifique.

Mansour, A., Fox, C. A., AkIL, H., \& Watson, S. J. (1995). Opioidreceptor mRNA expression in the rat CNS: Anatomical and functional implications. Trends in Neurosciences, 18, 22-29.

Matthews, R. T., \& German, D. C. (1984), Electrophysiological evidence for excitation of rat ventral tegmental area dopamine neurons by morphine. Neuroscience, 11, 617-625.

OLDS, M. E. (1979). Hypothalamic substrate for the positive reinforcing properties of morphine in the rat. Brain Research, 168, $351-360$.

Pettit, H. O., Ettenberg, A., Bloom, F. E., \& КоOB, G. F. (1984). Destruction of dopamine in the nucleus accumbens selectively at- 
tenuates cocaine but not heroin self-administration in rats. Psychopharmacology, 84, 167-173.

Phillips, A. G. (1984). Brain reward circuitry: A case for separate systems. Brain Research Bulletin, 12, 195-201.

Phillips, A. G., \& Le Piane, F. G. (1980). Reinforcing effects of morphine micro-injection into the ventral tegmental area. Pharmacology, Biochemistry \& Behavior, 12, 965-968.

Robson, L. E., Paterson, S. J., \& Kosterlitz, H. W. (1983). Opiate receptors. In L. L. Iversen, S. D. Iversen, \& S. H. Snyder (Eds.), Handbook of psychopharmacology (Vol. 17, pp. 13-80). New York: Plenum.

Simantov, R., ChIlders, S. R., \& SNYdeR, S. H. (1978). The opiate receptor binding interaction of $3 \mathrm{H}$-methionin enkephalin, an opiate receptor peptide. European Journal of Pharmacology, 47, 319-331.

STEIN, E. A., \& OlDS, J. (1977). Direct intracerebral self-administration of opiates in the rat. Society for Neuroscience Abstracts, 3, 302.

Stinus, L., Cador, M., \& Le Moal, M. (1992). Interaction between endogenous opioids and dopamine within the nucleus accumbens. In P. Kalivas \& H. H. Samson (Ed.), The neurobiology of drug and alcohol addiction (Annals of the New York Academy of Sciences, Vol. 654, pp. 254-273). New York: New York Academy of Sciences. Welzl, H., KuHN, G., \& Huston, J. P. (1989). Self-administration of small amounts of morphine through glass micropipettes into the ventral tegmental area of the rat. Neuropharmacology, 28, 1017-1023.

WINER, B. J. (1971). Statistical principles in experimental design. New York: McGraw-Hill

WiSe, R. A., \& BoZARTh, M. A. (1984). Brain reward circuitry: Four circuit elements "wired" in apparent series. Brain Research Bulletin, 12, 203-208.

Wise, R. A., \& Hoffman, D. C. (1992). Localization of drug reward mechanisms by intracranial injections. Synapse, 10, 247-263.

(Manuscript received August 7, 1995; revision accepted for publication March 11, 1996.) 